\title{
Trends in Physics
}

The General Conference of the European Physical Society is an exceptional event taking place only once every three years. The first was held in Florence in 1969, followed by Wiesbaden in 1972 and Bucharest in 1975. In September 1978, the conference will take place in the old city of York in Great Britain. The General Conferences are cultural events bringing together the various parts of physics, exploring the frontiers of physics and its links with other fields of science. Since the founding of EPS ten years ago, the general pattern of these conferences has changed. For York, it has been decided to associate eight specialized symposia in parallel and several plenary sessions devoted to talks on fast developing new subjects. Such a pattern is already used with success by national physical societies in national conferences.

The symposia are organized by the EPS Divisions and ACAPPI (Advisory Committee on Applied Physics and Physics in Industry). Their topics have been chosen so as to favour interdisciplinarity; many topics are at the boundary of different domains, so that they are of interest for several Divi- sions, but in each case, one Division (or ACAPPI) has agreed to take the main initiative. Each symposium has its own scientific committee which fixes, in an autonomous way, its scientific programme and chooses its speakers. Several symposia call for contributed papers either in poster sessions or, in some cases, in oral presentations. It should be possible, in this way, to review in detail recent progress and to have specialized discussions in the various fields covered by the symposia. The following articles in this issue of Europhysics News describe these topics and give some insight into their significance.

The titles of the lectures of the plenary sessions together with the names of the lecturers are listed opposite. They correspond to very different fields in micro and macrophysics, in theory and experiment, in pure and applied physics. These lectures are intended not for the specialist, but for the physicist at large who wishes to be informed on new important advances in domains of physics distinct from his own.

The programme committee has also decided to add both in the parallel and in the plenary sessions, sessions and lectures devoted to physics and education and to physics and society. These are two important matters that concern EPS and physicists in general, not only the specialists in these fields, but all physicists whatever their main fields of activity. These topics also are presented below.

Such a programme should attract junior and senior physicists, those who are actively involved in a highly specialized field and those who wish to obtain a broad coverage. It will interest laboratories, institutions and universities all over Europe. We look forward to seeing in York several hundred physicists from the various European countries. In order to help especially young physicists to come to York, EPS will allocate grants out of its conference fund, and several national societies also have obtained funds to do the same. 'Trends in Physics', the 4th General Conference of EPS, will be a memorable event.

\section{P. Radvanyi}

(Chairman, International Programme Committee)

\section{Symposium B 1: Surface Physics}

Although contributions to our understanding of solid surfaces and interfaces were being made fifty years ago, it was not until the 1960's and the development of ultra high vacuum technologies (as a spin-off from the space programme) that environments for consistent and reproducible surface experimentation became available. During the past two decades, chemists, materials scientists and physicists have worked to establish the coherent interdisciplinary field of surface science. Initially the most rapid growth of the field was in North America, however a strong European effort developed through the 1970's leading this June to the first European Conference on Surface Science (ECOSS-1, Amsterdam) and in September to this Surface Physics Symposium during the 4th General EPS Conference.

Experimental progress in surface science relies on the development of techniques sensitive to the properties of a fraction of a monolayer $\left(10^{13}-10^{14}\right.$ atoms $\left.\mathrm{cm}^{-2}\right)$. The most powerful involve the scattering of low energy electrons, ions and neutral atoms and the application of intermediate energy $(40-100 \mathrm{eV})$ photoemission. A great deal of effort has ben expended to understand the physics of these techniques and much remains to be done before they can be fully effective; e.g. few measurements of cross sections $\Phi$ are available and the dependence of $\Phi$ (E) with solid matrix remains essentially unmapped. During the Symposium a selection of technique-related problems will be examined ranging from the theoretically tractable (but experimentally difficult) method of Rutherford back scattering of $\mathrm{MeV} \mathrm{He}^{+}$from surfaces, allowing very precise layer location, to the theoretically challenging and emerging study of molecular vibrations at surfaces. One entire session will be devoted to photoemission, not only because this is the most productive area of surface research at present, but because this conference will allow a rare opportunity to bring leading European exponents in the areas of synchrotron radiation and surfaces together in joint session to discuss future joint interests. Of additional value to surface scientists will be an exhibition to demonstrate existing and anticipated synchrotron radiation facilities in Europe.

Semiconductor surfaces and interfaces deserve a special place in the Symposium in part because they represent an area where theoretical understanding is probably outstripping experimentation, a growing problem nowadays in surface science. Whereas the theory of surface electronic states is well developed, experimental location and measurement of state densities has only been well established 